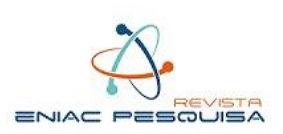

\title{
Diagnóstico de la Actividad Artesanal de la Palma como Desarrollo Económico en el Barrio El Carmen del Guamo - Tolima, Colombia
}

\author{
Diagnosis of the Artisanal Activity of La Palma as Economic Development in \\ the Barrio El Carmen del Guamo - Tolima, Colombia
}

Recebimento: 17/07/2021 - Aceite: 15/08/2021 - Publicação: 01/09/2021

Processo de Avaliação: Double Blind Review

Elizabeth Palma Cardoso

Institución de Educación Superior ITFIP, Colombia

epalma@itfip.edu.co

https://orcid.org/0000-0002-9650-8305

Ruth Erika Morales Lugo

Institución de Educación Superior ITFIP, Colombia

rmorales@itfip.edu.co

https://orcid.org/0000-0002-0754-3249

Yined Sánchez Ramirez

Institución de Educación Superior ITFIP, Colombia

ysanchez84@itfip.edu.co

https://orcid.org/0000-0003-4016-8564

Yessica Alexandra Yara Cuellar

Institución de Educación Superior ITFIP, Colombia

yyara62@itfip.edu.co

https://orcid.org/0000-0002-9863-2684

Robert Alexander Guzman

Magister en Educación, Especialista en Pedagogía, Contador Público.

Docente Investigador de la Institución de Educación Superior ITFIP

roguzman@itfip.edu.co

https://orcid.org/0000-0003-2608-8744

\section{RESUMEN}

El presente artículo de investigación tiene como propósito realizar un diagnóstico de la actividad artesanal de la palma como desarrollo económico en el barrio el Carmen del Guamo

- Tolima. El tipo de investigación es descriptivo, el método es el inductivo y el enfoque cuantitativo, las técnicas que se implementaron para llevar a cabo la investigación fueron como fuente primaria de recolección de datos la observación y las encuestas, en los cuestionarios que se elaboraron para las encuestas cada uno contenía 10 preguntas, esta técnica fue aplicada a los artesanos de la localidad para conocer los problemas que tienen en la comercialización de los productos que fabrican con palma real, además se recurrió a la fuente 


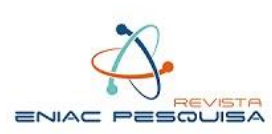

segundaria de recolección de datos donde se encontró información sobre el tema tratado en noticieros, revistas científicas, trabajos de investigación y textos. Con la información recolectada se evidenció que el principal problema que tienen los artesanos al vender sus productos es la falta de convenios.

Palabras Clave: artesanía, producción, economía familiar, cultura, tradición

\begin{abstract}
The purpose of this research article is to make a diagnosis of the artisanal activity of the palm as an economic development in the neighborhood of Carmen del Guamo - Tolima. The type of research is descriptive, the method is inductive and quantitative approach, the techniques that were implemented to carry out the research were as a primary source of data collection observation and surveys, in the questionnaires that were developed for the surveys each contained 10 questions, this technique was applied to the artisans of the locality to know the problems they have in the marketing of the products that they manufacture with real palm, in addition they resorted to the secondary source of collection of data where information on the subject was found in newscasts, scientific journals, research papers and texts. With the information collected, it was evident that the main problem that artisans have when selling their products is the lack of agreements.
\end{abstract}

Keywords: artisan, production, family economy, culture, tradition

\title{
1. INTRODUCCIÓN
}

Las artesanías que se elaboran con palma real son parte fundamental de la cultura del municipio del Guamo, ya que estas han contribuido a que los habitantes del municipio conserven sus costumbres no solo en los que ejercen esta actividad, sino que también en los que no la ejercen. La creación de las artesanías en el municipio generalmente es instruida de generación en generación en los hogares que producen estos artículos, es debido a ello que es común que varios o todos los integrantes de una misma familia se dediquen a realizar este arte para tener ingresos económicos.

Según (Bolivar, 2019) "La actividad de realizar artesanías con palma real ocupa uno de los principales renglones de trabajo de los habitantes del municipio el Guamo y esta es una de las materias primas más útiles en el arte de la tejeduría del sur del Tolima. El oficio va desde el corte y limpieza de la hoja de palma real, pasando por un proceso de tintes naturales, 


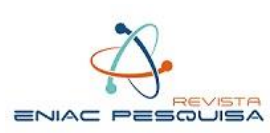

hasta el tejido hábil e ingenioso de artesanas que producen objetos utilitarios y decorativos muy apreciados por los turistas que visitan esta región”. (p. 39)

En el presente artículo se evidencia la importancia que tiene la actividad artesanal que se realiza con palma y también las dificultades que tienen los artesanos de la localidad del barrio el Carmen del Guamo Tolima, al momento de vender sus productos, artículos para uso personal o decoraciones de interiores con esta fibra natural. Así mismo, se busca dar recomendaciones que le ayuden a solucionar los inconvenientes y con ello, participar en la adecuada toma de decisiones para garantizar su competitividad y sostenibilidad empresarial. (Calderón, 2018; Batista y Guacari, 2018; Barrios, 2019; Reyes, Hernández, y González, 2019)

\section{DISCUSIÓN TEÓRICA}

\subsection{Actividad artesanal}

Según (Ruíz \& Pacheco, 2014) “..., la actividad artesanal es el núcleo del sistema y adquiere importancia en la medida que es una actividad social, económica y cultural local con identidad propia, que elabora mecanismos de adaptación fundamentada en sus capacidades."

Esta teoría sirve en este trabajo de investigación, ya que dan a conocer que la actividad artesanal le brinda una identificación especial al lugar donde se realiza, además de que con el paso del tiempo las actividades artesanales se van adhiriendo al contexto social, económico y cultural del municipio y por último que ayuda a las familias en la parte económica.

\subsection{Actividades artesanales que se realizan en el Guamo}

Como afirman el (DANE, 2020)“Las actividades artesanales que se realizan en la zona urbana del Guamo son la tejeduría y la sombrerería” (p 074).

El (Ministerio de desarrollo economico, 1989) plantea que:

La sombrerería es una actividad que hace parte de la tejeduría y se elabora especialmente con fibras vegetales, esta puede emplear varias técnicas, pero la que más se destaca es el cruce a mano para fabricar directamente el producto o para realizar trenzas que se unen atreves de la costura, al especialista en esta actividad se le denomina sombrerero y por otra parte el tejido o tejeduría es una actividad en el que se entrecruzan uno o más hilos o fibras con el uso de agujas o 
con las manos, los materiales que más se utilizan es el fique, la lana, las cerdas, crines, palma y pelo, el especialista se denomina tejedor. (p 19-23).

La tejeduría es una actividad que realizan varios habitantes del barrio el Carmen del Guamo con palma real, en donde se entrelazan con las manos las hojas de esta especie de palma y se elaboran las trenzas o también llamados cortes, los cuales se constituyen por diez brazadas que equivalen a 12 metros y la sombrerería en el municipio se encarga de unir con la máquina de coser los cortes fabricando una amplia gama de sombreros y demás artículos.

\title{
2.3. Palma real
}

Según Bernal \& Galiano (2013) afirman que:

\begin{abstract}
La attalea butyracea es conocida con el nombre de palma real en el departamento de Amazonas, Caquetá, Casanare, Cesar, Córdoba, Cundinamarca, Guainía, Huila y Tolima, aunque a lo largo del territorio colombiano es conocida con diferentes nombres como canambo, corozco de marrano, corozco de puerco, corozco de vaca, corúa, curúa, palma de corúa, cuesco, palma de cuesco, curumuta, corozo, palma dulce, palma de ramo, palma rial, sebón, yagua, además de que es conocida en el país principalmente con el nombre de palma de vino.(p 166)
\end{abstract}

Este concepto es muy importante debido a que, expone el nombre científico de esta especie de palma y los diferentes nombres que tiene en cada uno de los departamentos de Colombia, conocer los nombre que se recibe la palma en el territorio colombiano permite que se pueda recolectar una mayor cantidad de información.

\subsubsection{Tejidos de la palma.}

(Santos, 2020) propone que "El tejido de la palma es un proceso que se realiza para de fabricación de artículos que se enseña de generación en generación por los artesanos y por demás habitantes que tienen conocimiento sobre ella".

La teoría expuesta anteriormente, dio a conocer que el tejido que se realiza con la palma es una costumbre que no solo se transmite por los artesanos que elaboran productos con esa materia prima, sino también, por personas que tienen conocimiento de la manera como se realizan estas artesanías. 


\subsubsection{Tipos de tejido con palma en el Guamo}

Cocomá Reyes afirma que:

Para la elaboración de artesanías en el municipio del Guamo, hay diversos tipos de tejidos con palma que se utilizan para la fabricación de los productos artesanales, implementando solamente la palma real se pueden producir el corte palma real liso, corroncho, calado y el llamado argolla, además hay tejidos que se realizan empleando la palma real y palma de coco o palma de coco únicamente, estos son el corte liso palma de coco, petaquiado, cruzado, corte en cordón, peine y el denominado eme, con estos tejidos se pueden forjar tapetes, abanicos, pavas, bolsos, abanicos, pie de camas, manilla, adornos y demás accesorios. (pp 25-29)

A partir de lo anteriormente planteado, se concluyó que no todos los artesanos elaboran un mismo tejido, sino que elaboran una variedad de tejidos en el municipio del Guamo y también que se requiere por lo general más de un tipo de tejido para la elaboración de los productos artesanales y para hacer estos productos sean llamativos.

\subsubsection{Artesano}

Como expresa (Quiroa, 2010) “..., es la persona que realiza un trabajo manual y realiza piezas que son totalmente exclusivas, personalizándolas de acuerdo con los gustos de sus clientes, las piezas que fabrican siempre van a diferente, ya que, los procesos no están estandarizados".

Esta teoría permite identificar que las artesanías que elaboran los artesanos se fabrican de acuerdo a las preferencias del comprador además de que estos productos siempre van a poseer alguna diferencia debido a que los procesos que realizan no son exactamente los mismos.

\subsubsection{Costumbre}

Desde el punto de vista de (Raffino, 2020) "La costumbre es un conjunto de prácticas que realiza un individuo o una comunidad y se convierte en algo característico de la comunidad [...] las costumbres se reflejan en las danzas folclóricas, gastronomía, artesanías, música, ritos religiosos y en festividades ".

Del concepto anterior, se determina que la costumbre es una acción repetitiva que puede tener mucha importancia en una comunidad, ya que, estas pueden hacer que una población elabore leyes y además muestra que la enseñanza de las costumbres se transmite de generación en generación en las sociedades. 


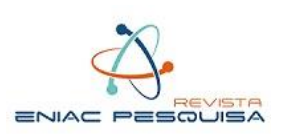

\subsubsection{Desarrollo económico}

Como afirma (Gómez Carrión, 2010) "El desarrollo económico es el resultado del crecimiento económico y se observa a través de las transformaciones y cambios estructurales en el sistema productivo" ( $\mathrm{p} 2$ ).

La teoría anterior, sirvió de guía para la investigación, ya que dio a conocer que el desarrollo trae cambios estructurales en sistema productivo y también cambios en la parte económica.

Es por ello, que para la actividad artesanal de este estudio, es importante el uso de erramientas virtuales, el cual, se a convertido en un elemento clave para el desarrollo económico específicamente del sector productivo, su implementación y uso incrementa la competitividad y tiene efectos positivos en el crecimiento económico, integración social y sostenibilidad ambiental. (Ceballos, Mejía \& Arango Medina, D. 2019, p. 65). Así como también, da soluciones a las diferentes problemáticas dentro del contexto empresarial (Barragán y Escobar 2017, p. 36). Y a su vez, esto permite un crecimiento financiero en toda entidad (Guzmán et al., 2020, p. 16)

\section{MATERIALES Y MÉTODOS}

Este artículo es resultado de una investigación de tipo descriptiva, ya que a través de la percepción de los artesanos del barrio el Carmen se conocieron los problemas que cuentan al vender sus productos en el mercado.

Con los resultados obtenidos mediante la fuente primaria de recolección de información se dieron posibles soluciones a las inconvenientes que tenían los artesanos al momento de comercializar los productos para así contribuir a que esta actividad siga permaneciendo en el municipio.

El método es inductivo, debido a que esta investigación permitió conocer los problemas que tenían los artesanos de manera individual y después se unieron todas las opiniones, para determinar los problemas generales de todo el sector de estudio y el enfoque es cuantitativo, debido a que, los cuestionarios que se utilizaron estaban constituidos por preguntas cerradas. 


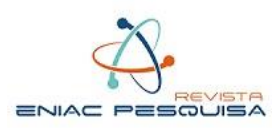

Para recolectar información suficiente para llevar a cabo el proyecto de investigación fue necesario acudir a la fuente primaria y a la segundaria, además se implementaron varias técnicas. De acuerdo con (Torres Jiménez \& Pérez Guerra, 2017) "En la realización de una investigación las técnicas que se implementen deben contribuir al logro de los objetivos que se han planteado".

La información se recolectó a partir de charlas y escritos proporcionados por los artesanos de la localidad del Carmen, las técnicas que se implementaron fueran observación y la encuesta.

Para tener soporte del tema tratado se recurrió al internet en donde a través de diversos sitios web se sustentó la investigación, la información que se recolectó provenían de textos, noticieros, revistas y trabajos de grado de varios autores.

\section{RESULTADOS}

La Figura 1 indica que, el 6\% de los artesanos consideran que la cualidad que más poseen las artesanías que elaboran en el barrio el Carmen con palma real es que son llamativas; el 17\% afirman que la característica que poseen mayoritariamente los artículos del sector es que cuentan con una amplia variedad y el $77 \%$ expresan que el atributo que poseen es que están constituidos por una buena calidad. 


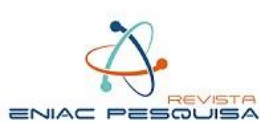

\section{Figura 1}

De los siguientes enunciados ¿cuál considera que es la cualidad que más poseen las artesanías con palma real que produce el sector?

\section{Cualidad que poseen las artesanías}

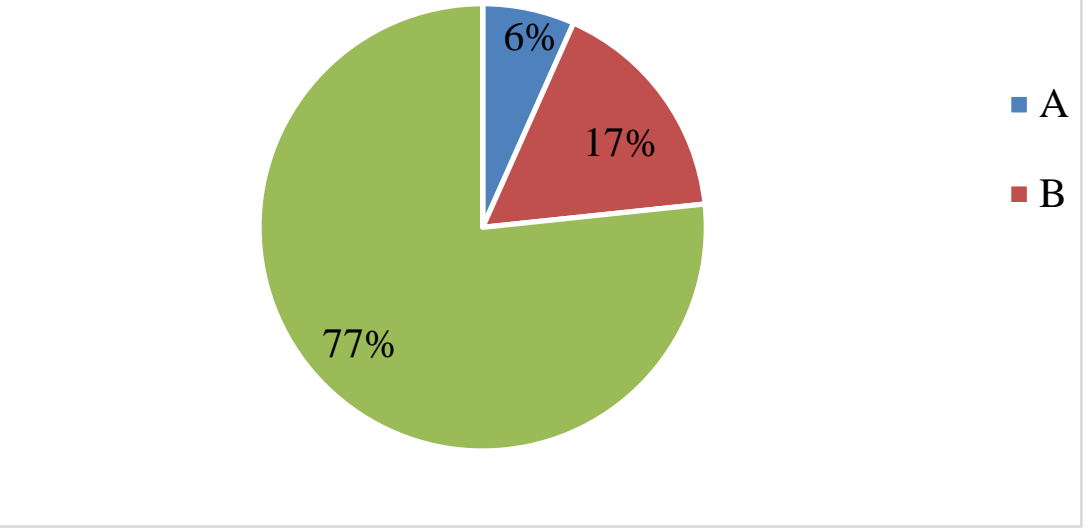

Fuente: Elaboración de los autores.

En la Figura 2 se observa que, el 97\% elaboran más el tejido con palma real reconocido comúnmente como corte de palma real liso y el 3\% elaboran principalmente con esta fibra natural el corte denominado corroncho. 


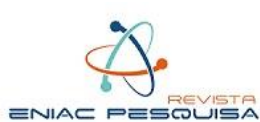

\section{Figura 2}

De los siguientes enunciados ¿cuál considera que es la cualidad que más poseen las artesanías con palma real que produce el sector?

Cuál de los siguientes tejidos usted elabora más?

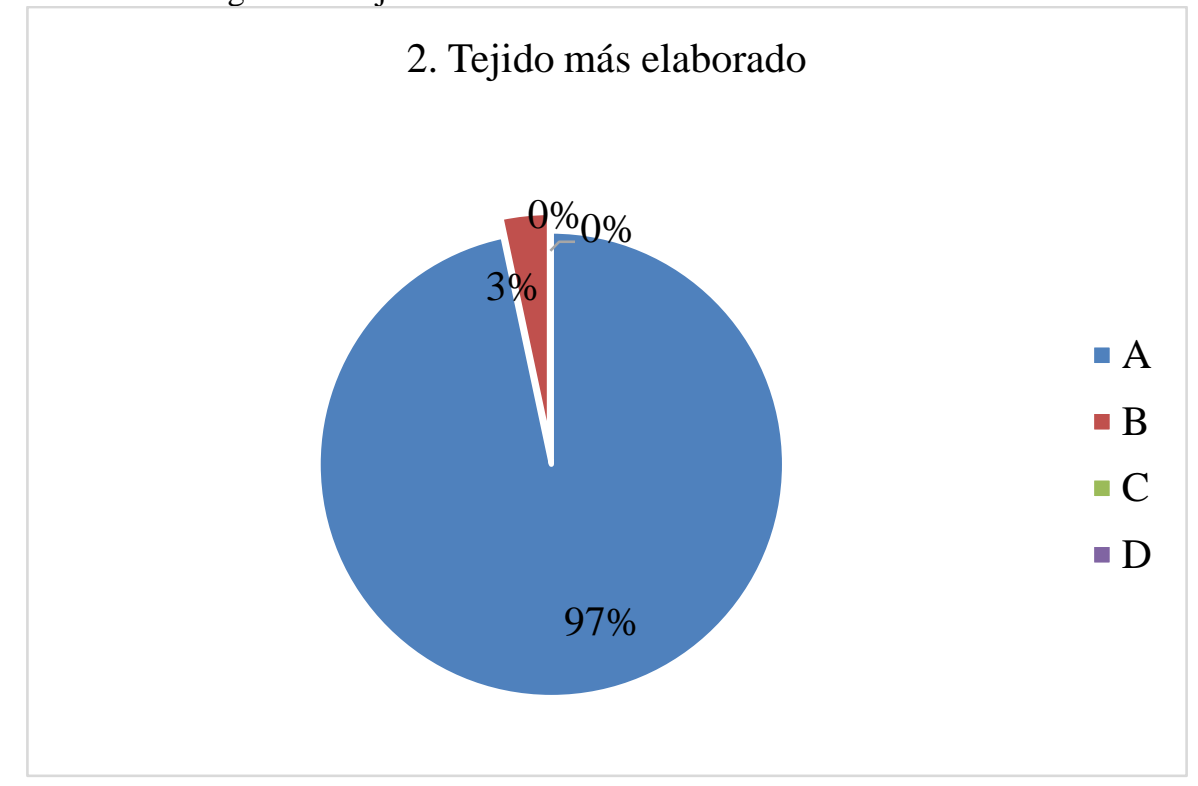

Fuente: Elaboración de los autores.

La Figura 3 muestra que, del 100\% de los artesanos, el $83 \%$ fueron instruidos a realizar artesanías en palma real por sus familias, el $14 \%$ de los encuestados aprendieron a fabricar los productos artesanales por sus vecinos y el 3\% expusieron que elaborar los artículos con esa fibra natural se las enseño un amigo. 


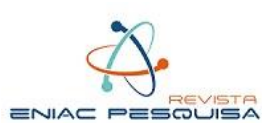

\section{Figura 3}

Quién le enseño a usted a elaborar artesanías con palma real?

\section{Enseñanza}

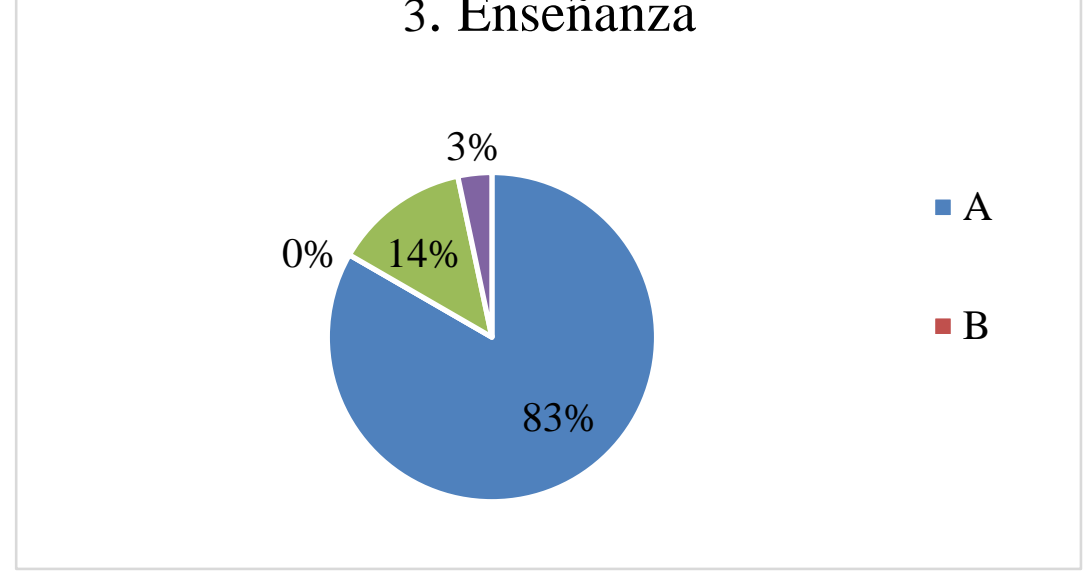

Fuente: Elaboración de los autores

En la Figura 4 se puede evidenciar que, el 20\% de los artesanos manifiestan que, la comercialización de las artesanías del barrio el Carmen se ven alterados por la falta de una marca, el 20\% consideran que, la venta de los productos artesanales se encuentra afectados al no realizarles publicidad y el $60 \%$ de los encuestados afirman que los perjudica es no contar con convenios con empresas. 

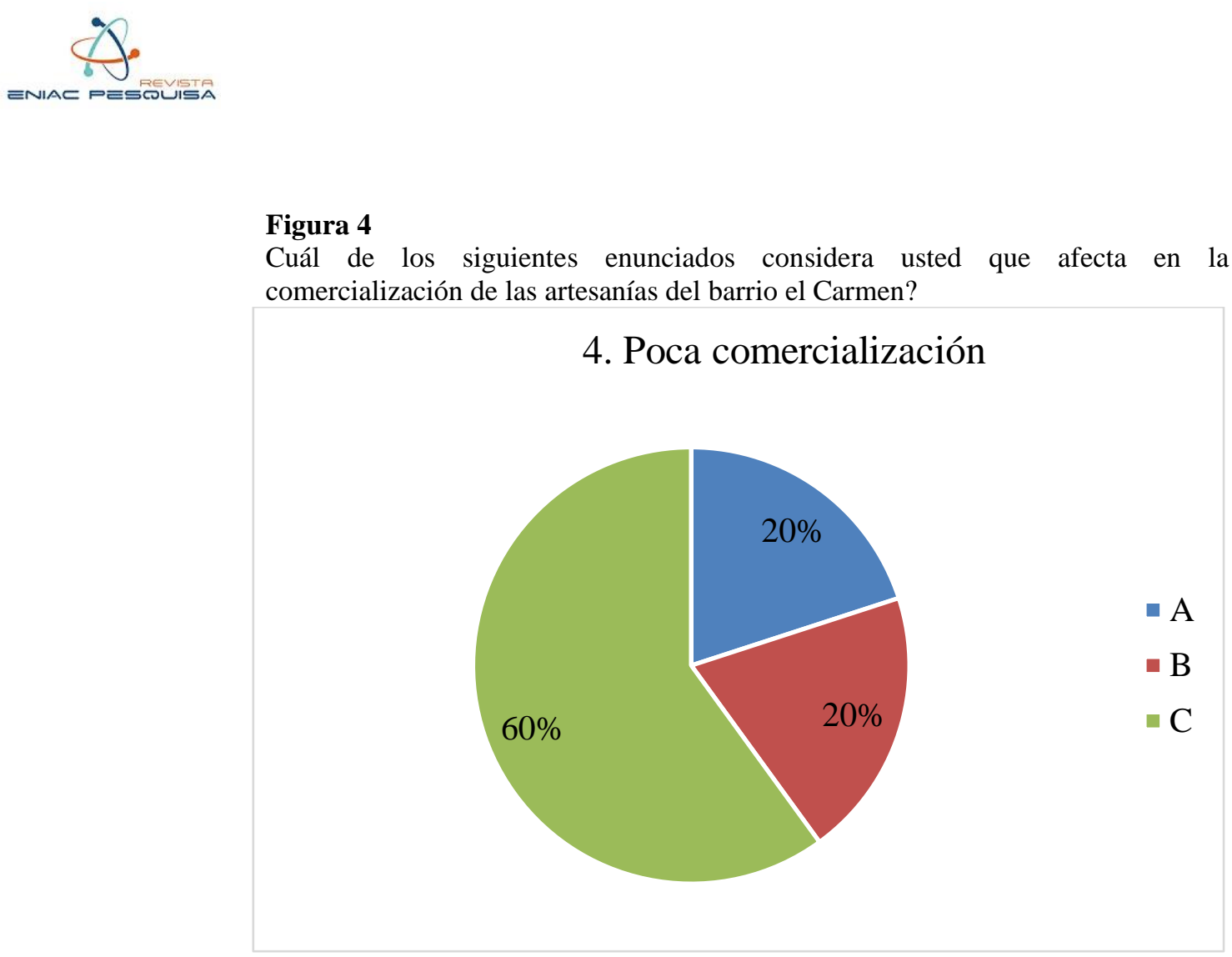

Fuente: Elaboración de los autores

En la Figura 5 se logra observar que, el 90\% de los artesanos del barrio el Carmen, les gustaría hacer parte de una marca que creara el municipio del Guamo para comercializar las artesanías, el 7\% no les interesa hacer parte y el 3\% de las personas de los encuestadas expresaron que hacen parte de una asociación y ya cuentan con una marca. 


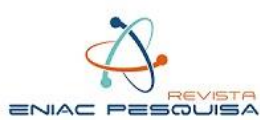

\section{Figura 5}

Si el municipio del Guamo creará una marca para comercializar las artesanías que se elaboran en el barrio el Carmen ¿ Le interesaría hacer parte?

\section{Creación de una marca}

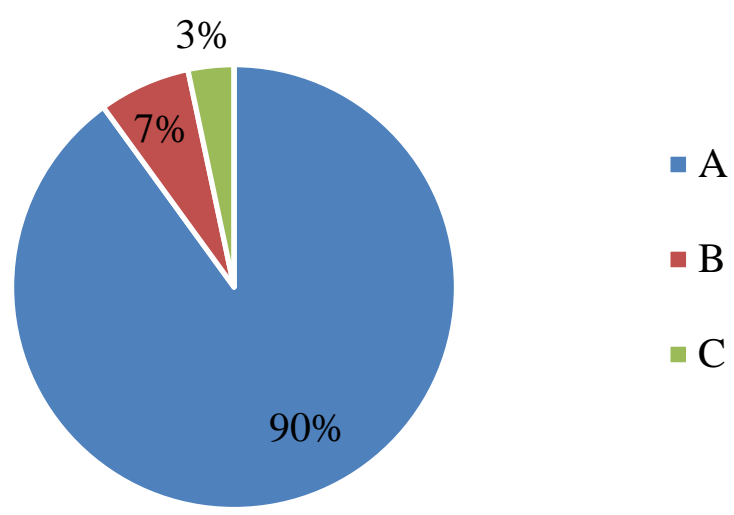

Fuente: Elaboración de los autores

Em la Figura 6 se observa que el 13\% de las personas dicen que algunas veces la falta de una marca afecta la comercialización de las artesanías e influyen en el bajo precios que tienen en el mercado, el $10 \%$ opinan que no las afecta la falta de una marca y el $77 \%$ de los artesanos considera que si los afecta no poseer una marca ya que esto generaría que los productos fueran más reconocidos y aumentaran el precio. 


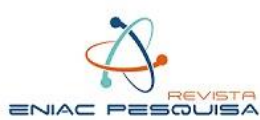

\section{Figura 6}

La falta de una marca propia afecta la comercialización de sus productos e influye directamente en que los productos tengan un bajo precio en el mercado?

\section{Falta de una marca propia}

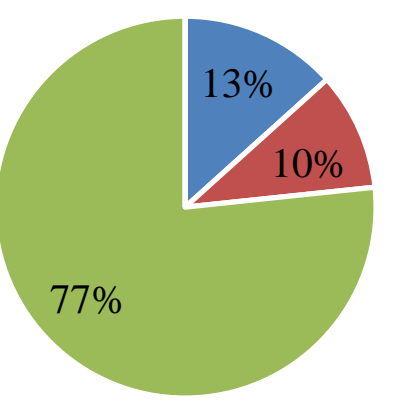

$\because \mathrm{A}$

- B

$\square \mathrm{C}$

Fuente: Elaboración de los autores

En la Figura 7 se demuestra que, el 90\% de los artesanos expresan que no vender los productos directamente en otros municipios o tener convenios con empresas genera que las artesanías que fabrican en el sector no sean tan conocidas por habitantes de otros municipios y el $10 \%$ de los artesanos afirman que no vender sus artículos en diversos lugares no ha producido que personas de otros lugares desconozcan los productos. 


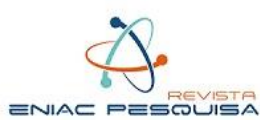

\section{Figura 7}

Considera que no vender directamente sus productos en otros municipios o hacer convenios con empresas han provocado que muchas personas de otros pueblos no tengan conocimiento de que el municipio se elaboran este tipo de artesanías?

\section{Desconocimiento del producto}

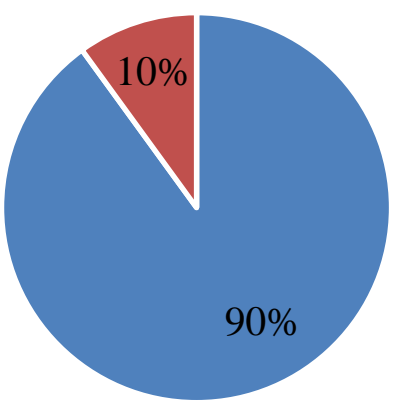

- A

- B

Fuente: Elaboración de los autores

La Figura 8 muestra que del 100\% de los encuestados, el 54\% de los artesanos recibe menos de cien mil pesos mensualmente por la elaboración y comercialización de sus artesanías, el $13 \%$ obtienen ingresos mensuales de 100.000 a 150.000 mil pesos, el $13 \%$ perciben ingresos mensuales entre 150.000 a 200.000 mil pesos y el $20 \%$ obtienen más de 200.000 mil pesos por la fabricación y comercialización de los productos. 

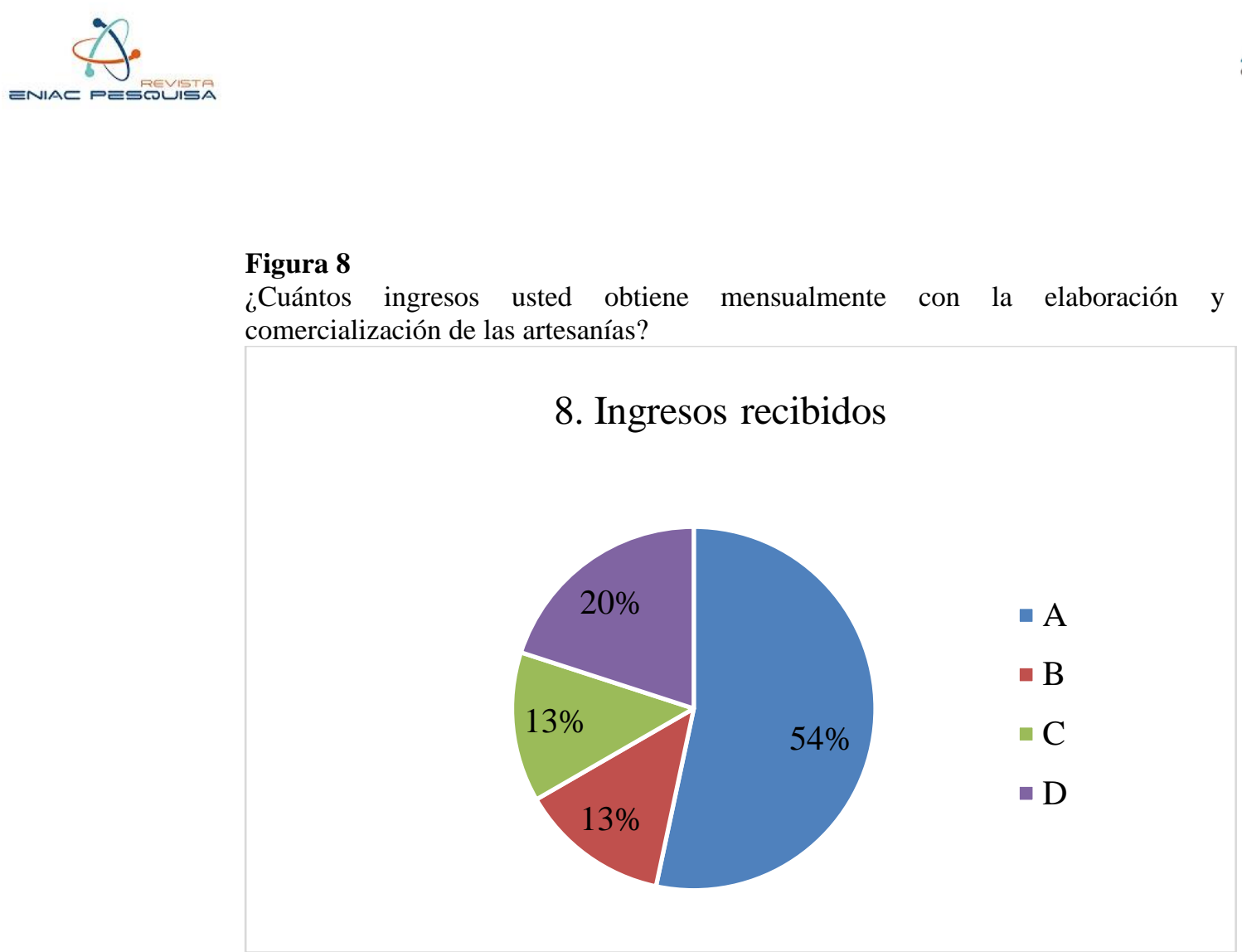

Fuente: Elaboración de los autores

La Figura 9 indica que el 73\% de los artesanos obtienen la mayoría de sus ingresos mensualmente por realizar la actividad artesanal, el 10\% de los encuestados respondieron que sus ingresos mensuales no provienen principalmente por ejercer esta actividad y el 17\% dicen que algunas veces perciben la mayor entrada de ingresos por hacer esta actividad. 


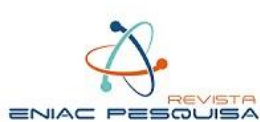

\section{Figura 9}

¿La mayoría de sus ingresos mensuales son obtenidos por esta actividad artesanal?

\section{Origen de sus ingresos}

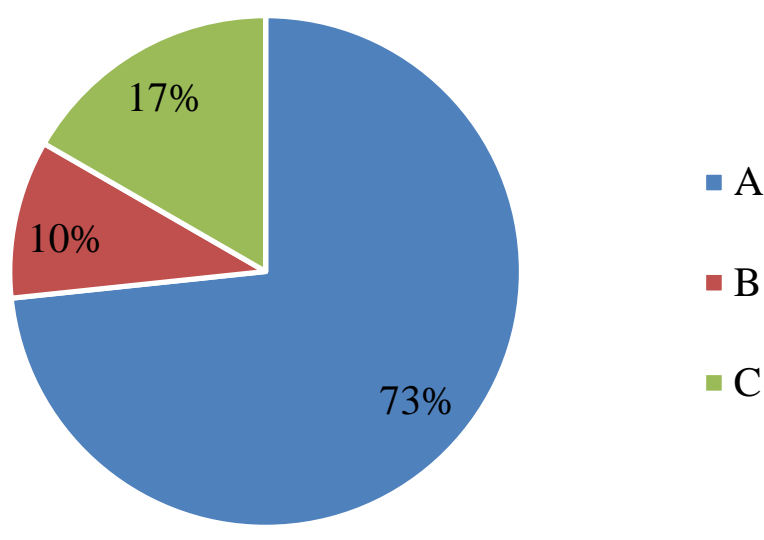

Fuente: Elaboración de los autores

En la Figura 10 se logra evidenciar que, el $20 \%$ de los artesanos han pensado en abandonar esta actividad artesanal, y el $80 \%$ de las personas encuestadas respondieron que nunca dejaran de ejecuta este arte así no les genere el beneficio económico que ellos esperan obtener.

\section{Figura 10}

¿Ha pensado dejar esta actividad artesanal porque no le genera la rentabilidad económica que espera obtener?

\section{Dejar de realizar la actividad}

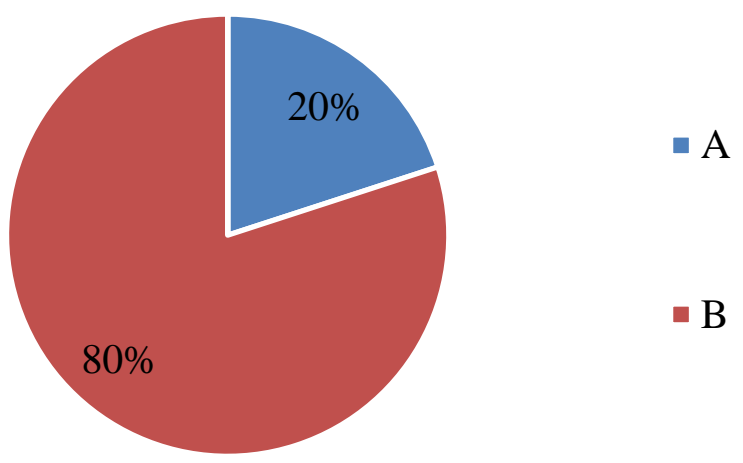

Fuente: Elaboración de los autores 


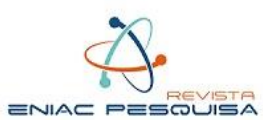

En el gráfico de la Figura 11 se muestra que, el $44 \%$ de los artesanos consideran que realizar esta actividad les ha contribuido a mejorar su calidad de vida, el 39\% de las personas encuestadas opinan que ejercer la labor no les ha ayudado a restablecer la condición de vida y el 17\% de los encuestados dicen que llevar a cabo este arte solo en temporadas les ha servido a aumentar sus ingresos y también ha colaborado a su salud mental.

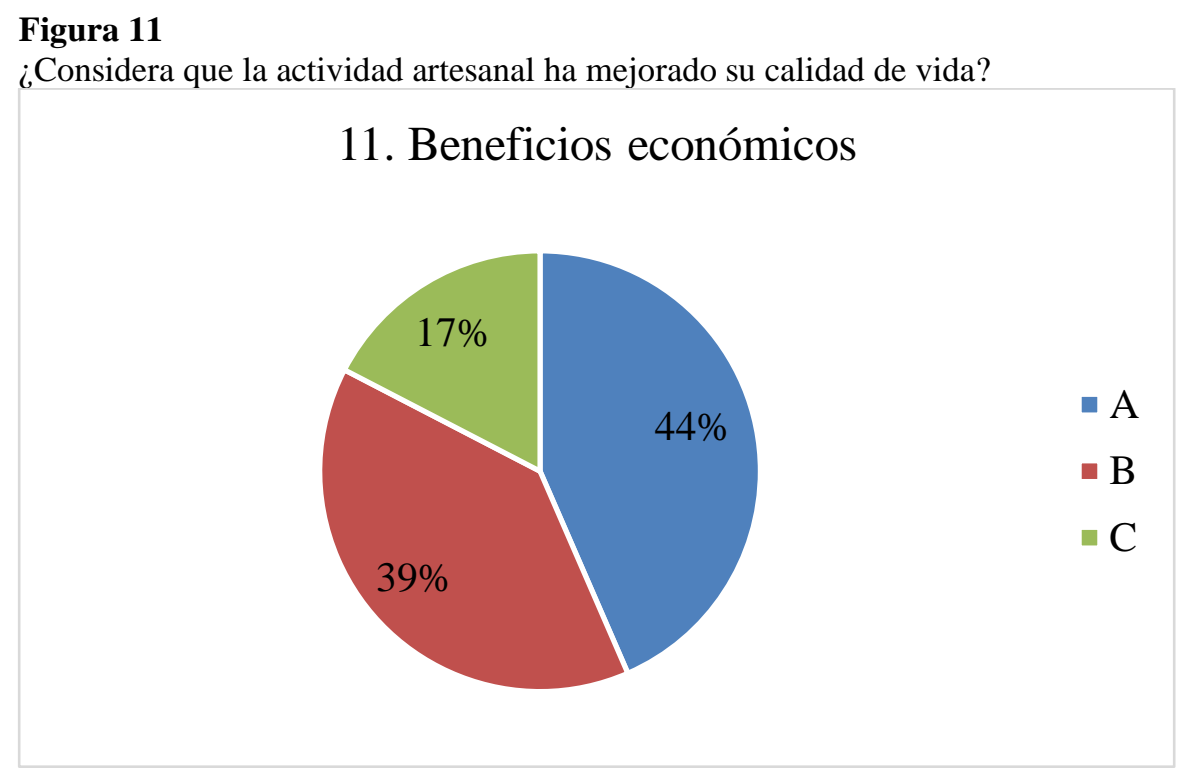

Fuente: Elaboración de los autores

En la Figura 12 se evidencia que, del 100\% de los artesanos, el $78 \%$ de los encuestados expresan que si en el barrio el Carmen dejaran de ejercer esta actividad artesanal con palma real ellos perderían las costumbres que le han transmitido y el $22 \%$ de las personas perciben que al desaparecer este arte no perderían sus hábitos. 


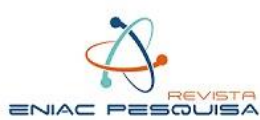

\section{Figura 12}

Para usted la actividad artesanal si dejará de existir en el barrio el Carmen ¿Usted perdería parte de sus costumbres?

\section{Dejar la actividad artesanal}

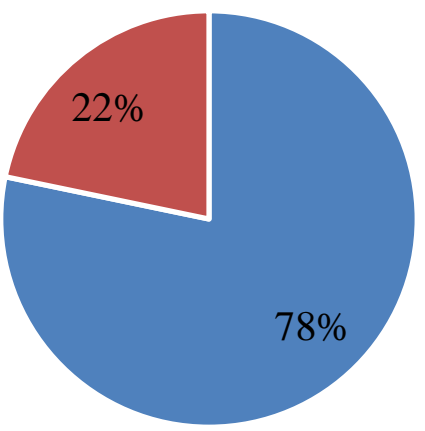

- A

$\because \mathrm{B}$

Fuente: Elaboración de los autores

En la Figura 13 se refleja que, el 57\% de las personas que se les aplicó la encuesta afirman que los productos que se elaboran con fibras naturales diferentes a la palma real obstaculizan que las artesanías que se realizan en el barrio el Carmen con palma sean más competitivas en el mercado y el $43 \%$ opinan que los artículos que fabrican con otros elementos no dificultan la comercialización de las artesanías, debido a que, cada pieza que producen los artesanos tienen un uso especial. 


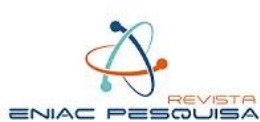

\section{Figura 13}

¿Para usted las artesanías (abanicos, sombreros, bolsos) que realizan con otras fibras naturales obstaculizan que los productos con palma real sean más competitivos en el mercado?

\section{Competencia de los artículos}

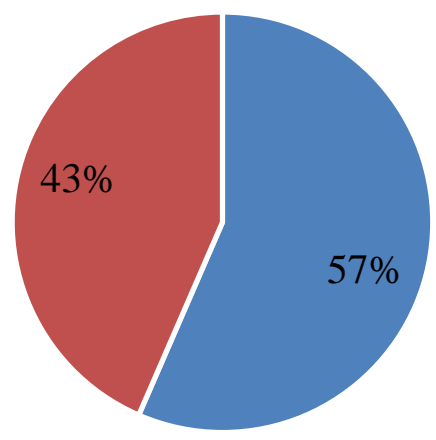

Fuente: Elaboración de los autores

La Figura 14 muestra que, del 100\% de los encuestados, el 43\% de los artesanos tiene un lugar fijo en el municipio en donde venden los productos que realizan y el $57 \%$ de las personas a las que se le aplicó la encuesta respondieron que no cuenta con un lugar determinado en el que comercialicen sus artesanías, esto produce que para venderlas tengan que desplazarse a diversos lugares hasta que encuentren algún cliente que las adquiera.

\section{Figura 14}

¿Tiene un lugar definido en el municipio del Guamo donde usted comercializa las artesanías?

\section{Comercialización}

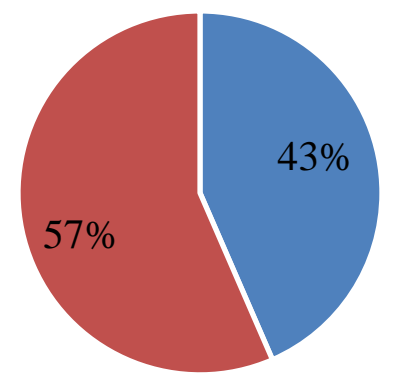

Fuente: Elaboración de los autores 


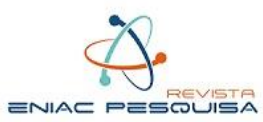

Se puede analizar que el $13 \%$ de los artesanos alcanzan a suplir las necesidades con lo que obtienen mensualmente al realizar esta actividad, mientras que, el 65\% de ellos, no logran suplir todas sus obligaciones, debido a ello, tienen que apoyarse ejecutando otra labor, y el $22 \%$ de los encuestados respondieron que algunas veces el recurso económico que obtienen por llevar a cabo este arte, les alcanza para cumplir con los compromisos adquiridos.

\section{Figura 15}

¿Con lo que obtiene mensualmente con esta actividad le alcanza para suplir todas las necesidades que tiene?

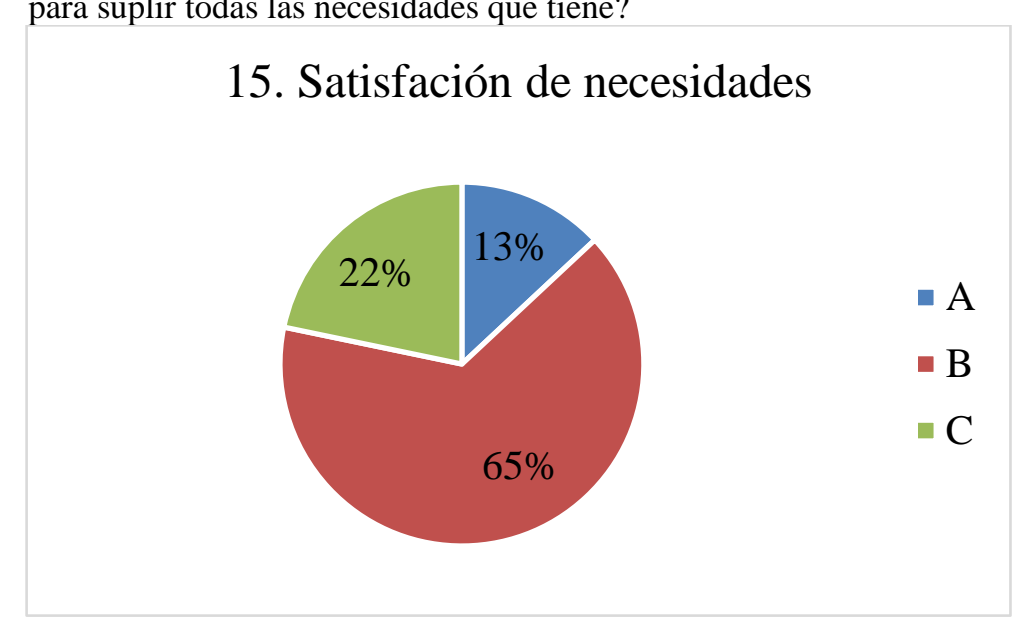

Fuente: Elaboración de los autores

Se evidencia en el gráfico anterior, que del 100\% de las personas que se les aplicó el cuestionario, el 17\% respondió que la actividad artesanal en sus hogares era realizada por los adultos mayores, el $48 \%$ de los artesanos afirman que los integrantes que ejecutan la actividad son mujeres y el 35\% de los encuestados expresaron que esa actividad en su familia la ejercen todos los miembros, desde los niños, hasta los adultos mayores. 


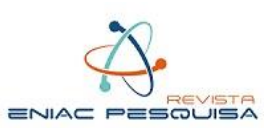

\section{Figura 16}

¿Qué integrantes de su familia ejercen esta actividad

\section{Ocupación de la actividad}

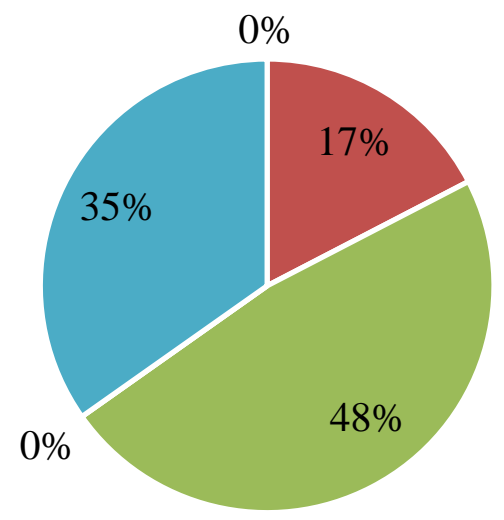

- A

- B

$\because \mathrm{C}$

- D

$\because \mathrm{E}$

Fuente: Elaboración de los autores

La Figura 17 permite evidenciar que, el 70\% de los artesanos expresaron que han tenido que enseñarles a algunos de los integrantes de su familia a fabricar artículos con palma real para que la economía de sus hogares incrementará y el 30\% de los encuestados afirmaron que no se le han instruido a elaborar este arte para ayudar con la economía familiar.

\section{Figura 17}

Para ayudar con la economía de su hogar ha tenido que enseñarles a algunos de miembros de su hogar este arte?

\section{Mejoramiento de la economía familiar}

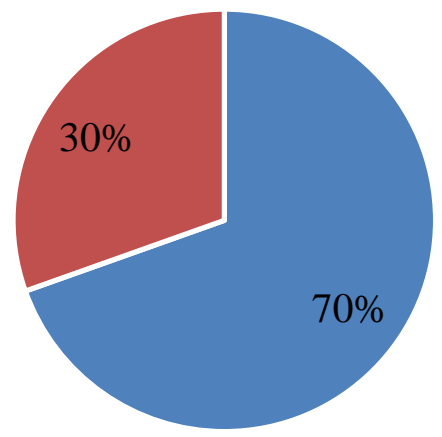

Fuente: Elaboración de los autores 


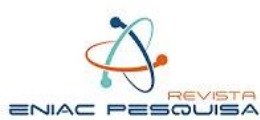

La Figura 18 muestra que, el $91 \%$ de los artesanos expresan que si el gobierno prohibiera que se siga ejerciendo la actividad artesanal con la palma real se verían gravemente afectados sus ingresos y el $9 \%$ de las personas encuestadas consideran que sus recursos económicos no estarían seriamente alterados al no continuar realizando este arte.

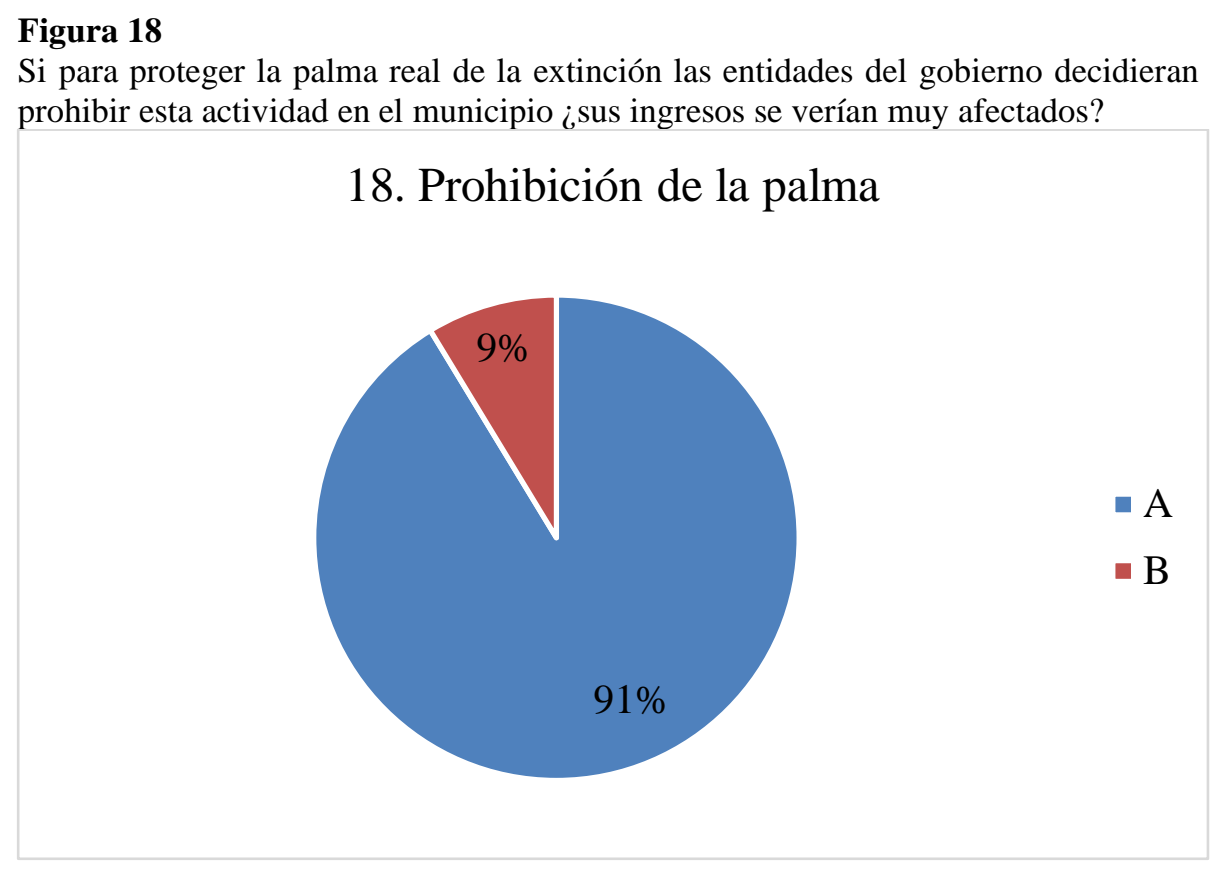

Fuente: Elaboración de los autores

El gráfico indica que, el $22 \%$ de las personas encuestadas han pensado en dejar esta actividad artesanal, ya que, no tienen un cliente que le compre los productos frecuentemente y el $78 \%$ de los artesanos afirman que nunca dejaran esta labor a si no cuenten con un comprador habitual. 


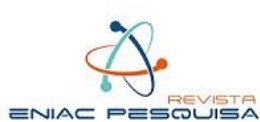

\section{Figura 19}

¿Ha pensado dejar esta actividad artesanal en algún momento porque no tiene una empresa o cliente frecuente que le compre los productos?

\section{Dejar la actividad artesanal}

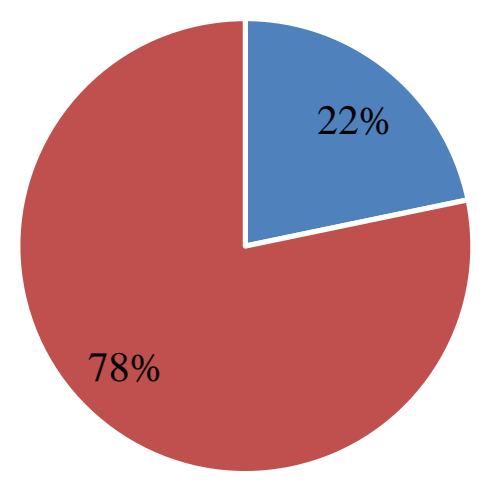

Fuente: Elaboración de los autores

La Figura 20 se puede observar en que, el 57\% de los artesanos afirman que obtienen ingresos constantes todo el año por la venta de los productos que fabrica y el $43 \%$ de los encuestados expresan que no adquieren beneficios todo el año con la comercialización de sus artesanías.

\section{Figura 20}

¿Los ingresos que obtiene por la venta de sus artesanías son constantes todo el año?

\section{Variación de los ingresos}

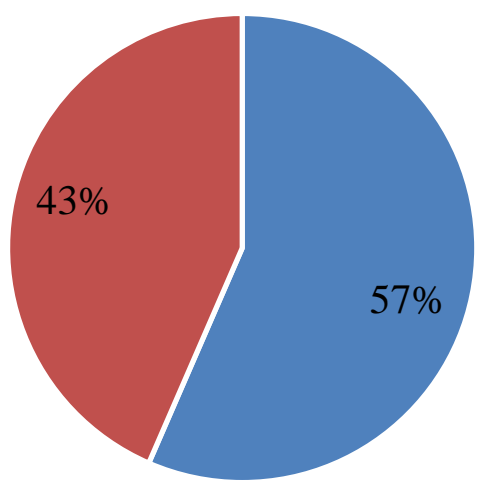

$\because \mathrm{A}$

$-\mathrm{B}$

Fuente: Elaboración de los autores 


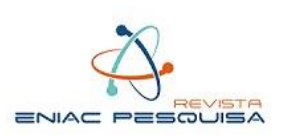

Las encuestas realizadas a los artesanos del barrio el Carmen, permitieron conocer la importancia de esta actividad para la tradición del municipio y para la parte económica de las familias artesanas, además, se logró evidenciar los problemas que ellos poseen al momento de comercializar sus productos y la falta que les hace tener convenios con empresas del municipio y de municipios aledaños, por otra parte, al no contar con una marca producen que no reconozcan el lugar donde fabrican los productos las personas.

Por otra parte, también se pudo conocer la perspectiva que tienen los artesanos sobre los productos que elaboran ellos y los demás artesanos de la localidad, en donde la mayoría percibe que la cualidad que es más notoria en las artesanías del sector es que poseen una excelente calidad, también se evidencio que muchas personas que ejecutan esta actividad dependen económicamente de ella.

Elaborar la encuesta fue muy útil, porque se recogió información importante que sirvió para conocer el desarrollo de esta actividad, además, permitió confirmar el pensamiento que se tenía sobre porque les enseñaban los artesanos este arte a sus hijos, el cual era de que esta actividad la enseñaban comúnmente en los hogares para tener más entradas de efectivo y también para que esta actividad artesanal que realizan con palma real no se acabara y así esta costumbre que han tenido los habitantes del municipio en el especial del barrio el Carmen siga perdurando por mucho tiempo.

\section{DISCUSIÓN}

Un primer hallazgo encontrado es el documento de Serrano (2019), la información que se estaba almacenada en ese artículo plasmaba que el $69 \%$ de los artesanos del departamento del Tolima son mujeres y el 39\% lo conforman los hombres, al aplicar las encuestas se logró afirmar que las personas del sexo femenino ejercían más la actividad artesanal con palma real.

Con los resultados obtenidos se logró evidenciar lo que expreso Hernández \& Martínez (2012) en donde dieron a conocer que la actividad artesanal es un hábito que las personas realizan, lo dicho anteriormente se comprobó mediante los cuestionarios aplicados, ya que la mayoría de los artesanos consideran que ejercen esta actividad ya que, hacen parte de su tradición y la de sus familias. 


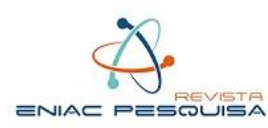

El trabajo realizado permitió dar cumplimiento al objetivo de la investigación, en donde se logró conocer la importancia económica y cultural que tiene para los artesanos del barrio el Carmen elaborar productos con palma real, además se logró comprender las dificultades que poseen los tejedores y sombrereros al comercializar sus artesanías en el mercado.

Por otra parte, este proyecto contribuyó a que los artesanos reflexionaran sobre el valor que ha tenido este arte en sus vidas, en las de sus familias y en la de todos los habitantes del municipio, también los motivo a seguir ejerciendo esta labor, debido a que percibieron que la actividad artesanal que ellos llevan a cabo genera interés en las demás personas.

Por último, con la implementación de este proyecto se logró incentivar a que los jóvenes y niños a que, continuaran desempeñando esta ocupación, dándoles más oportunidades para que mejoren su condición de vida y también, que esta tradición perdure por muchos años más en el municipio del Guamo.

\section{CONSIDERACIONES FINALES}

En este estudio, se logró concluir que la falta de una marca propia en el sector, hace que los productos que se fabrican con palma real, sean menos conocidos en la región y que además, esto les genera que sus productos al no ser reconocidos en otros lugares diferentes al municipio del Guamo, hacen que el precio que obtienen por vender estas artesanías sea más bajo, lo que le afecta directamente en sus ingresos.

Por otra parte, con la información recopilada, se conoció que los artesanos del barrio el Carmen, no cuentan con un lugar específico para vender sus artesanías, lo que les genera que se tengan que trasladar a otros lugares, hasta encontrar alguna empresa o algún cliente a quienes le puedan ofrecer sus artículos.

Por último, con las encuestas aplicadas a los artesanos también se logró conocer un poco sobre la economía de ellos, donde se podía observar que las personas que se dedicaban solamente a tejer los cortes son los que obtienen menos beneficios económicos, ya que ellos no cuentan con los recursos suficientes para transformar la materia prima en productos terminados, es debido a esto que estas familias han tenido que dedicarse a realizar diferentes actividades al mismo tiempo para poder mejores sus ingresos, en cambio que las personas que 


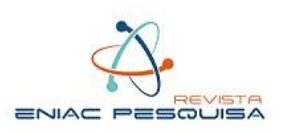

se dedican a terminar todo el proceso de estos artículos son personas capacitadas y además cuentan con la maquinaria suficiente para realizarlos y por ese motivo estos artesanos solo dependen de la actividad artesanal ya que al realizarla les suple todas sus necesidades que ellos presentan.

\section{REFERENCIAS}

Adames Giraldo, L. (2019). Tejeduría con palma de Wérregue. Obtenido de https://repositorio.unbosque.edu.co/bitstream/handle/20.500.12495/2958/Adames_Giraldo_Li na_2019.pdf? sequence $=1$ \&isAllowed $=\mathrm{y}$

Andrade, V. (2017). Impacto del aprovechamiento de hojas sobre las poblaciones de palma amarga (Sabal. Obtenido de https://repositorio.unal.edu.co/bitstream/handle/unal/59526/37086384.2017.pdf?sequence=1\# page $=30$ :

Barragán, F. y Escobar, S. (2017) Modelo resignificación para incentivar el uso de las TIC en la práctica pedagógica en Coreducación Revista INNOVA ITFIP, 1 (1), 36-46

Barrios, J. (2019). Impacto de la Innovación en la Competitividad de las Empresas del Sector Agroindustrial, en el Espinal-Tolima - Colombia. Revista Innova ITFIP. 4 (1). 7-20

Batista, A., y Guacari, W. (2018) gestión empresarial y competitividad en las mipymes del centro comercial nueva Colombia de la ciudad de Cartagena., Revista INNOVA ITFIP, 3 (1), 75-81

Batlle, J. (Septiembre de 2017). equipos y talentos. Obtenido de ¿Qué es vender?: https://www.equiposytalento.com/tribunas/open--out/que-es-vender

Bembibre, C. (Junio de 2009). Definición de Ingresos. Obtenido de https://www.definicionabc.com/economia/ingresos.php

Bembibre, C. (Marzo de 2013). DefiniciónABC. Obtenido de Definición de Enseñar: https://www.definicionabc.com/general/ensenar.php

Bernal, R., \& Galeano, G. (2013). Academia. Obtenido de Palma de vino (Attalea butyracea). En: R. Bernal \& G. Galeano (Eds.) Cosechar sin destruir. Aprovechamiento sostenible de palmas colombianas: https://www.academia.edu/26133496/Palma_de_vino_Attalea_butyracea_En_R_Bernal_and_ G_Galeano_Eds_Cosechar_sin_destruir_Aprovechamiento_sostenible_de_palmas_colombian as

Betancourt Céspedes, M. J., \& Capera López, L. J. (2020). Plan De Negocio Para La Comercialización De Productos A Base De Cerámica Y Palma Real A. Obtenido de https://repository.ucc.edu.co/bitstream/20.500.12494/32826/4/2020_plan_negocio_ceramica.p df 
Bolivar, L. (10 de Febrero de 2019). issuu. Obtenido de Estado del Arte Artesanias del Tolima : https://issuu.com/leonorbolivar/docs/estado_del_arte_del_tolimaa_final

Calderón Saque, E. A. (2018). Educación económica y financiera, una solución a la exclusión. Revista Innova ITFIP, 3(1), 44-51. Recuperado a partir de http://www.revistainnovaitfip.com/index.php/innovajournal/article/view/38

Ceballos, O. I., Mejía Castellanos, L. A., \& Arango Medina, D. (2019). AUDITORIA DE USABILIDAD DE HERRAMIENTAS IMPLEMENTADAS EN PLATAFORMAS VIRTUALES PARA OFERTAR SERVICIOS CON RESPONSABILIDAD SOCIAL. Revista Innova ITFIP, 5(1), 64-77. Recuperado a partir de http://www.revistainnovaitfip.com/index.php/innovajournal/article/view/56

Cocomá Reyes, C. (Junio de 2010). Uso y manejo de la palma real (attalea butyracea) para la elboracion de artesanias en el departamento del Tolima. Obtenido de https://repository.javeriana.edu.co/bitstream/handle/10554/8506/tesis464\%20\%282\%29.pdf?s equence $=3 \&$ is Allowed $=\mathrm{y}$

Cruz Gonzáles, M. (2007). La actividad artesanal como atractivo complementario al desarrollo turístico sostenible de la provincia de Casma- Ancash. Obtenido de https://dspace.unitru.edu.pe/bitstream/handle/UNITRU/7757/Tesis\%20Maestr\%c3\%adaX\%20

$\% 20 \mathrm{Mar} \%$ c3\%ada\%20de1\%20Carmen\%20Cruz\%20Gonz\%c3\%a1les.pdf?sequence=1\&isAll owed $=\mathrm{y}$

DANE. (2020). Economía naranja. Obtenido de https://www.economianaranja.gov.co/media/xlxnv51c/cuarto-reporte-naranja.pdf

Díaz Pava, M. F., Palma Cardozo, E., Varón Giraldo, O., Gutiérrez Delgado, D. M., Rodríguez López, W. D., Guzmán, R. A., Sabogal Barreto, R. (2019). Conociminetos contables básicos para comerciantes al por menor del municipio de Icononzo - Tolima. Espinal: Instituto Tolimense de Formación Tecnica profesional - ITFIP. Obtenido de https://itfip.edu.co/investigacionfeac/wp-content/uploads/2021/02/LIBRO-ICONONZO.pdf

Einforma Colombia. (2017). Asociación de artesanos de la palma real del Guamo - Tolima Asopalguamo trabajo recuperado de Obtenido de https://www.einforma.co/servlet/app/portal/ENTP/prod/ETIQUETA_EMPRESA/nif/1700455 713900000\#

Giraldo, V. (23 de Agosto de 2019). rockcontent. Obtenido de Descubre qué es la publicidad, para qué sirve y cómo influye en la sociedad: https://rockcontent.com/es/blog/publicidad/

Gobernación del Tolima. (2020). Turismo. Obtenido de https://www.tolima.gov.co/tolima/informacion-general/turismo/1906-municipio-de-elguamo?_cf_chl_jschl_tk_=fa2f94e6d30af80a611a5bf42ce40ba6ae20cf14-1624253871-0ARtW_Z9MqC9D0KdE--kXg_VGTckH83sezro06H1ivVHSolubE3U_rIVuB_J-rHTinB3_DHiM7r0g08vEi9la4yTopp-C

Gómez Carrión, G. Y. (Junio de 2010). CONCEPTO SOBRE EL DESARROLLO ECONÓMICO. Obtenido http://biblioteca.usbbog.edu.co:8080/Biblioteca/BDigital/65859.pdf 


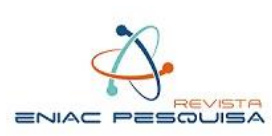

Gómez Chancafe, R. S. (2020). Producción artesanal de "paja palma macora" para el desarrollo del turismo vivencial en ciudad Eten, región Lambayeque. Obtenido de https://repositorio.udl.edu.pe/bitstream/UDL/423/1/GomezChancafe_Tesis\%20AT.pdf

Guzmán, R., Caycedo, M., Perdomo, J. y Rengifo, J. (2020). Evaluación del Sistema de Control Interno de un Proceso de Tesorería en la Institución Educativa Técnica Gabriela Mistral. En Guzmán, R., Palma, E., y Morales, R., (2020). (Eds.). El Revisor Fiscal y la evaluación del control interno. Edición 1. Editorial Instituto Tolimense de Formación Técnica Profesional ITFIP. (pp. 11-30)

Hernandez, A., \& Martinez, N. (27 de Septiembre de 2012). Plan de mercadeo para la comercialización de los productos artesanales "DIARA" en la ciudad de Villavicencio. Obtenido de slidehare: https://es.slideshare.net/huelgosangelica/proyecto-de-grado-artesanas2012-0928

Lopez Aponte, C. (Mayo de 2018). BUAD. Obtenido de Artesanías e identidad en la comunidad cestera de san Luis Atolotitlan, puebla: https://repositorioinstitucional.buap.mx/handle/20.500.12371/8080

Martinez, M. A. (17 de Septiembre de 1997). Artesanías de Colombia s.a. unidad de diseño. Obtenido http://www.artesaniasdecolombia.com.co/propiedadintelectual/comunidades/artesaniascolombia-sombreros-iraca-suaza-huila.pdf

Merino Gordillo, M. A. (2 de Febrero de 1987). Decreto 258. Obtenido de https://ar.unesco.org/sites/default/files/colombia_decreto_258_02_02_1987_spa_orof.pdf

MinCultura. (06 de Junio de 2009). La Ley 1185 de 2008 y el Decreto 763 de 2009. Obtenido de https://mincultura.gov.co/areas/fomento-regional/noticias/Paginas/2009-07-06_24383.aspx

Ministerio de desarrollo economico. (1989). Listado General de oficios ertesanales. Obtenido de Centro de Investigación y Documentación "C $\mathrm{E} \quad \mathrm{N} \quad \mathrm{D}$ A $\mathrm{R}$ ": https://artesaniasdecolombia.com.co/trazaartesanal/documentos/artesanias-colombia-listadooficios.pdf

Minjusticia . (2020). Sistema unico de informacion normativa. Obtenido de DECRETO 2291 DE 2013 : http://www.suin-juriscol.gov.co/viewDocument.asp?ruta=Decretos/1432019

Minjusticia. (2019). Sistema unico de informacion normativa. Obtenido de LEY 36 DE 1984: http://www.suin-juriscol.gov.co/viewDocument.asp?ruta=Leyes/1592386

Montes de Oca, J. (20 de Julio de 2015). Economipedia. Obtenido de Crédito: https://economipedia.com/definiciones/credito.html

Morales, A. A., \& Serrano, D. (2017). Diagnóstico departamental del sector artesanal chocó. Obtenido de https://repositorio.artesaniasdecolombia.com.co/bitstream/001/4103/1/INSTD\%202017.\%2042.pdf

Moreno Ávila, G., \& Hernández Rojas, L. E. (Junio de 2018). La teoría del comercio justo como factor de desarrollo económico en el municipio de Planadas -Tolima. Revista Innova ITFIP, $\quad$ Volumen $\quad 2, \quad 54 \quad-\quad 60 . \quad 6$ Obtenido de http://revistainnovaitfip.com/index.php/innovajournal/article/view/26/37 
Naranjo Acosta, W. G. (Diciembre de 2020). La velocidad del dinero en Colombia (2005 2020). Revista innova ITFIP, volumen 7, 42-56. Obtenido de http://revistainnovaitfip.com/index.php/innovajournal/article/view/100/198

Peiró, R. (04 de Junio de 2020). Enconomipedia. Obtenido de Calidad: https://economipedia.com/definiciones/calidad-2.html

Quiroa, M. (10 de Enero de 2010). Economipedia. Obtenido de Artesano: https://economipedia.com/definiciones/artesano.html

Raffino, M. E. (5 de Septiembre de 2020). Concepto.de. Obtenido de Concepto de costumbre: https://concepto.de/costumbre/

Raffino, M. E. (28 de Mayo de 2020). Concepto.de. Obtenido de Concepto de cliente: https://concepto.de/cliente/

Reyes, G., Hernández, O., y González, F., (2019) Liderazgo comunitario y su influencia en la sociedad como mejora del entorno rural, Revista INNOVA ITFIP. 5 (1). 15-27.

Rivadeneira, S. (04 de Junio de 2020). Artesanas del Guamo preparan un catálogo virtual para vender sus productos el Día del Sombrero Típico Tolimense. Obtenido de elolfato: https://www.elolfato.com/region/artesanas-del-guamo-preparan-un-catalogo-virtual-paravender-sus-productos-el-dia-del

Roldán, P. N. (23 de Noviembre de 2016). Economipedia. Obtenido de Marca: https://economipedia.com/definiciones/marca.html

Ruíz, C. A., \& Pacheco, J. C. (2014). El estado y la actividad artesanal. Revista artifices, 1-41. Obtenido

de https://artesaniasdecolombia.com.co/Documentos/Contenido/14835_revista_artifices-primeraedicion.pdf

Santos, A. (14 de Agosto de 2020). Pasos sencillos para elaborar una cesta de palma. Obtenido de https://www.chiapasparalelo.com/trazos/cultura/2020/08/pasos-sencillos-paraelaborar-una-cesta-de-palma/

Serrano , D. (2019). Características sociodemográficas de la población artesanal. Obtenido de https://artesaniasdecolombia.com.co/Documentos/Contenido/32007_tolima.pdf

Sevilla Arias, A. (29 de Enero de 2016). Economipedia.com. Obtenido de Precio: https://economipedia.com/definiciones/precio.html

Sierra Galindo, M. J., Martínez Carazo, P., \& García Chávez, I. E. (2020). Caracterización de 4 productores de artesanías derivadas de la Palma de Vino en el municipio de Galeras, Sucre: un estudio de caso. Obtenido de https://www.researchgate.net/profile/PiedadCarazo/publication/347460590_Caracterizacion_de_4_productores_de_artesanias_derivadas_ de_la_Palma_de_Vino_en_el_municipio_de_Galeras_Sucre_un_estudio_de_caso/links/60370 e5d299bf1cc26ebfaaf/Caracterizacion-de-4-p

Tirado Orrillo, W. M., \& Ugaz Uriarte, J. T. (2016). Actividades Primarias de la Cadena de valor en los productores de sombrero de palma del Distrito de Bambamarca, para la exportación a la ciudad de Pamplona - España, 2017. Obtenido de https://repositorio.upn.edu.pe/bitstream/handle/11537/9987/Tirado\%20Orrillo\%20Winy\%20 


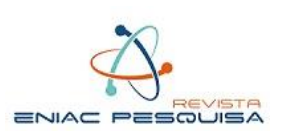

Melissa\%20Mawi\%2c\%20\%20Ugaz\%20Uriarte\%20Jessica\%20Tatiana.pdf?sequence=1\&isA llowed=y

Torres Gomez, J. L. (4 de Febrero de 2019). Blogger. Obtenido de La fuente primaria: técnica de la encuesta: http://lisandrotorresitfip.blogspot.com/

Torres Jiménez, E. D., \& Pérez Guerra, L. A. (Diciembre de 2017). Factores que inciden en la productividad investigativa. Revista Innova ITFIP, 105-111. Obtenido de http://www.revistainnovaitfip.com/index.php/innovajournal/article/view/19/25

Trujillo, E. (01 de Febrero de 2021). Economipedia. Obtenido de Convenio: https://economipedia.com/definiciones/convenio.html 